\title{
Gamificação e imersão como elementos de engajamento para simuladores: uma proposta de aplicação em realidade virtual para a Odontologia
}

\author{
Allan A. Tori \\ Interlab/EP/USP \\ São Paulo, Brasil \\ atori@usp.br
}

\author{
Romero Tori \\ Interlab/EP/USP \\ São Paulo, Brasil \\ tori@usp.br
}

\author{
Fatima L. S. Nunes \\ $L A p I S$ - EACH/USP e Interlab - EP/USP \\ São Paulo, Brasil \\ fatima.nunes@usp.br
}

\begin{abstract}
Resumo-Os simuladores em realidade virtual são alternativas para ensinar técnicas das áreas da saúde, no entanto, podem falhar em manter o engajamento e motivação do aprendiz. Elementos importantes para engajamento como a gamificação, podem ser fundamentais para contornar essa falha. Aliado a isso, a imersão visual, favorecida pelo uso de dispositivos Headmounted display(HMD), que promovem a estereoscopia com amplitude do campo de visão e agência da cabeça, tem se mostrado eficaz no aprendizado em simuladores e serious games. Além da imersão visual, é possível inserir a imersão interativa, com o uso de rastreamento do olhar e das mãos, por meio da qual o aluno poderá interagir com o mundo virtual de forma mais ativa e natural. A contribuição deste trabalho será estudar a gamificação e imersão como elementos capazes de promover o engajamento em simuladores. Como estudo de caso, um simulador será gamificado para a área da odontologia, assim como elementos de imersão, que também serão acrescentados neste simulador.
\end{abstract}

Index Terms-serious game, gamificação, simulador, realidade virtual.

\section{INTRODUÇÃO}

Atualmente o termo gamificação vem se tornando cada vez mais comum no meio educacional e corporativo. A "gamificação" é definida como o uso de elementos de design de jogos em um outro contexto [1]. Os alunos de hoje cresceram jogando videogames e é evidente que a popularidade desses jogos está aumentando [2], portanto a aplicação desses elementos em diversos contextos da vida das pessoas torna-se importante, como já é feito em sistemas digitais gamificados no meio educacional, que são mais conhecidos como serious games.

Os serious games ou jogos sérios se referem a aplicações interativas que vão muito além do mercado de videogames, incluindo assim, treinamento, análise, visualização, simulação, educação, saúde e terapia [3]. Os serious games considerados visualmente imersivos, são aqueles que fazem o uso de tecnologias que promovem a estereoscopia com amplitude do campo de visão e agência da cabeça, comumente presentes nos dispositivos HMD para a realidade virtual(RV).

Por essas razões, este trabalho busca contribuir com uma análise da real contribuição de dois elementos: a gamificação e a imersão. Para esse estudo, um simulador para a odontologia será gamificado e um experimento também será realizado com os usuários finais, que são os alunos de Odontologia.

\section{MotivaÇÃo}

Num primeiro momento, uma revisão sistemática foi feita para resgatar e analisar os serious games validados para a área da saúde. Dessa forma foi possível elencar elementos de jogos mais utilizados e como esses sistemas gamificados foram avaliados. Os resultados, em sua grande maioria, apontaram benefícios expressivos com o uso de elementos de jogos na educação e treinamentos em diversas áreas da saúde. Engajamento e motivação foram os fatores mais favorecidos e que estão diretamente ligados ao processo de aprendizado.

Outro fator importante para o processo de aprendizado foi a imersão. Um experimento foi realizado, figura $1 \mathrm{em}$ azul, com o simulador VIDA Odonto [4] e a imersão visual, com auxilio do HMD, e o retorno háptico, possibilitado com o uso de um dispositivo háptico (phantom omni), foram avaliados e seus benefícios foram evidenciados.

Nesses estudos preliminares com serious games e simuladores imersivos, percebeu-se uma área ainda não muito explorada, que são os serious games imersivos para aprendizagem de técnicas de risco, como a técnica de anestesia odontológica. A proposta aqui apresentada, pretende contribuir com uma análise e estudo de dois elementos: gamificação e a imersão. Para essa análise, a gamificação do simulador VIDA Odonto [4] será realizada, o qual irá permitir alunos de graduação a aprender e praticar técnicas da odontologia, mas com elementos de jogos e de interação ainda mais intuitivos.

\section{Trabalhos CorRelatos}

Alguns trabalhos de serious games em realidade virtual para a saúde já foram propostos na literatura e em todos foram notadas, a partir de métodos de avaliação, vantagens do sistema gamificado imersivo [5] [6]. Um sistema para treinamento médico com imagens em raio-x [5], por exemplo, ou até mesmo sistemas para reabilitação física, que muitas vezes são desmotivantes por serem monótonos e repetitivos [6], se beneficiaram da gamificação e imersão. 


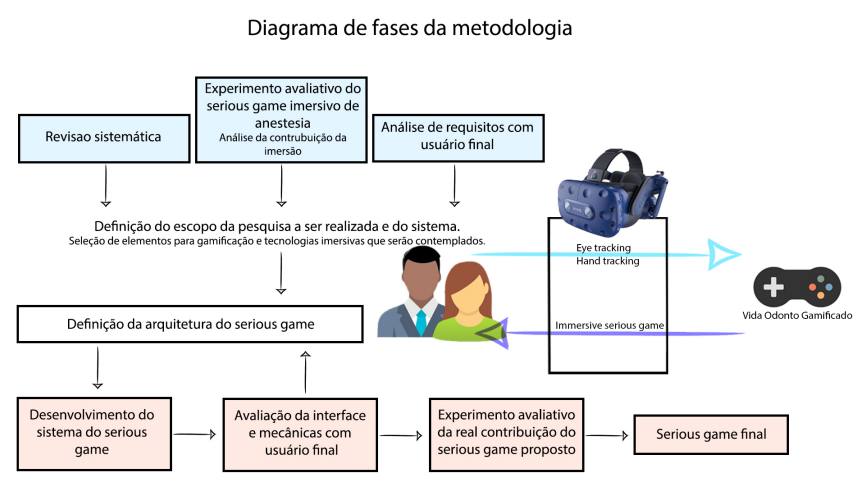

Figura 1. Representação da metodologia empregada no processo de desenvolvimento do serious game.

As formas como esses sistemas foram avaliados variaram, mas em muitos deles foram utilizados os questionários de pósteste [5], uma vez que esse instrumento permite avaliar se o conhecimento do assunto ou técnica proposta foi adequadamente assimilado pelo usuário, ou se houve de fato eficácia na reabilitação do usuário.

\section{SoluÇÃo Proposta}

A figura 1 mostra um diagrama com a metodologia que está sendo empregada neste trabalho. Os estudos realizados a partir da revisão sistemática e experimento com o VIDA Odonto [4], proporcionaram dados importantes para a definição do escopo da pesquisa a ser realizada. A parte em azul (fase um da figura 1) engloba atividades já realizadas ou em andamento: a revisão sistemática sobre serious games da área da saúde e o experimento avaliativo da imersão com o VIDA Odonto como atividades já realizadas. A análise de requisitos está em andamento. Após a conclusão dessas atividades, o escopo da pesquisa e a definição da arquitetura do protótipo da gamificação serão realizados.

Ainda na figura 1, a parte em cor salmão indica as atividades finais (fase dois) em que a primeira versão do desenvolvimento da gamificação do VIDA Odonto terá a sua interface e mecânicas de jogos avaliadas em um primeiro experimento de usabilidade. Com os feedbacks desse experimento, eventuais alterações necessárias deverão refletir na redefinição da arquitetura e no desenvolvimento do protótipo.

Por fim, um experimento avaliativo da real contribuição da gamificação proposta com a intenção de avaliar se houve diferenças no desempenho do aprendiz ao usar versões diferentes do simulador que consideram ou descartam gamificação e imersão. Instrumentos adequados para verificação de engajamento serão pesquisados e aplicados, a fim de relacionar o desempenho do aprendiz com o nível de engajamento percebido pelo usuário.

O protótipo a ser estudado será desenvolvido com a engine "Unity" e o framework da "SteamVR" para desenvolvimento do gamificação com ferramentas de rastreamento do olhar e das mãos. Para a aplicação dos elementos de jogos, o design pattern Singleton [7] será empregado, assim como a orientação a eventos para controle dos comportamentos e feedbacks do sistema.

\section{Resultados Preliminares}

Com a revisão sistemática foi possível perceber que houve um crescimento no interesse pelos serious games nas áreas da saúde. Nos trabalhos selecionados percebeu-se que os elementos de jogos permitiram criar sistemas antes vistos como monótonos, para sistemas mais motivantes e eficientes. Os resultados das avaliações, empregadas para validação dos sistemas encontrados, foram positivas em sua grande maioria, assim como demonstrou o experimento, fase um, realizado com o simulador VIDA Odonto [4].

No experimento concluiu-se que a imersão visual foi fundamental para a melhora nos resultados dos alunos no procedimento de anestesia, contribuindo positivamente para a nossa hipótese de que a inclusão da imersão auxilia na realização de uma técnica de forma mais rápida e eficiente. Outros elementos como imersão interativa deverão ser avaliados durante o desenvolvimento do projeto.

\section{CONCLUSÃO}

Os resultados preliminares forneceram informações importantes de gamificação e imersão para a educação, que servirão de base para a pesquisa aqui proposta. A gamificação, na revisão sistemática, se mostrou eficiente em motivar os alunos no aprendizado, assim como a imersão visual tornou a experiência mais envolvente e eficiente.

O presente projeto pretende avaliar os elementos de gamificação e a imersão interativa como meios de aprimorar o engajamento percebido pelo usuário.

\section{REFERÊNCIAS}

[1] T. Brigham, "An introduction to gamification: Adding game elements for engagement," Medical reference services quarterly, vol. 34, pp. 471-480, 102015 .

[2] M. de Bie and L. Lipman, "The use of digital games and simulators in veterinary education: An overview with examples," Journal of Veterinary Medical Education, vol. 39, no. 1, pp. 13-20, 2012, pMID: 22433739. [Online]. Available: https://doi.org/10.3138/jvme.0511.055R

[3] V. Narayanasamy, K. W. Wong, C. C. Fung, and S. Rai, "Distinguishing games and simulation games from simulators," Comput. Entertain., vol. 4, no. 2, p. 9-es, Apr. 2006. [Online]. Available: https://doi.org/10.1145/1129006.1129021

[4] R. Tori, G. Z. Wang, L. H. Sallaberry, A. A. Tori, E. C. de Oliveira, and M. A. de AM Machado, "Vida odonto: Ambiente de realidade virtual para treinamento odontológico," Revista Brasileira de Informática na Educação, vol. 26, no. 02, p. 80, 2018.

[5] M. Süncksen, H. Bendig, M. Teistler, M. Wagner, O. J. Bott, and $\mathrm{K}$. Dresing, "Gamification and virtual reality for teaching mobile X-ray imaging," in 2018 IEEE 6th International Conference on Serious Games and Applications for Health (SeGAH), 2018, pp. 1-7.

[6] I. Afyouni, F. U. Rehman, A. Qamar, A. Ahmad, M. A. Rahman, S. Ghani, and S. Basalamah, "Gamifying hand physical therapy with intelligent 3d navigation," SIGSPATIAL Special, vol. 8, no. 1, p. 42-49, Jun. 2016. [Online]. Available: https://doi.org/10.1145/2961028.2961035

[7] E. Gamma, R. Helm, R. Johnson, and J. Vlissides, "Design patterns: Abstraction and reuse of object-oriented design," in ECOOP' $93-$ Object-Oriented Programming, O. M. Nierstrasz, Ed. Berlin, Heidelberg: Springer Berlin Heidelberg, 1993, pp. 406-431. 